\title{
On the Impact of Ultra-Wideband (UWB) on Macrocell Downlink of UMTS and CDMA-450 Systems
}

\author{
Bazil Taha Ahmed and Miguel Calvo Ramon
}

\begin{abstract}
Results of the analytical analysis to assess the effect of the ultra-wideband (UWB) emissions on the [universal mobile telecommunication system (UMTS) and code division multiple access systems (CDMA-450)] are presented. The (UMTS and CDMA450 systems) normalized range and normalized capacity degradation are evaluated. A free-space propagation model is used to calculate the UWB signal power that interferes with both mobile systems. It is shown that, for the case of a single UWB transmitter, the UMTS can easily tolerate UWB interference when the UWB equivalent

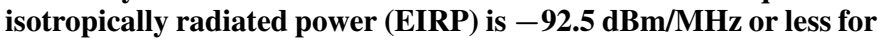
a distance between the UWB transmitter and the UMTS mobile of $1 \mathrm{~m}$ or higher. Also, it is shown that, for the case of multi-UWB transmitters, the UMTS can easily tolerate the UWB interference when the UWB EIRP is $-94.5 \mathrm{dBm} / \mathrm{MHz}$. For the single UWB transmitter case, the CDMA-450 downlink can tolerate UWB interference when the UWB power density is in the order of $-106 \mathrm{dBm} / \mathrm{MHz}$. For the case of multi-UWB transmitters, the power density that can be tolerated by the downlink of the CDMA450 system is in the order of $-108 \mathrm{dBm} / \mathrm{MHz}$.
\end{abstract}

Index Terms-Code division multiple access systems (CDMA450), macrocell normalized capacity, macrocell normalized range, ultra-wideband (UWB), universal mobile telecommunication system (UMTS).

\section{INTRODUCTION}

$\mathbf{T}$ HE FEDERAL Communications Commission (FCC) agreed in February 2002 to allocate $7.5 \mathrm{GHz}$ of spectrum for unlicensed use of ultra-wideband (UWB) devices for communication applications in the $3.1-10.6 \mathrm{GHz}$ frequency band. The move represented a victory in a long hard-fought battle that dated back decades. With its origins in the 1960s, when it was called time-domain electromagnetic, the UWB came to be known for the operation of sending and receiving extremely short bursts of radio-frequency (RF) energy. With its outstanding ability for applications that require precision distance or positioning measurements, as well as high-speed wireless connectivity, the largest spectrum allocation ever granted by the FCC is unique, because it overlaps other services in the same frequency of operation. Previous spectrum allocations for unlicensed use have opened up bandwidth dedicated to unlicensed devices based on the assumption that the operation is subject to the following two conditions.

Manuscript received June 21, 2006; revised November 15, 2007.

B. T. Ahmed is with the Escuela Politecnica Superior, Universidad Autonoma de Madrid, Madrid 28049, Spain (e-mail: bazil.taha@uam.es).

M. C. Ramon is with the European Telecommunications Standards Institute (ETSI), Universidad Politecnica de Madrid, Mardrid 28040, Spain (e-mail: miguel@gr.ssr.upm.es).

Digital Object Identifier 10.1109/TEMC.2008.919033
1) This device may not cause harmful interference. Harmful interference is defined as interference that seriously degrades, obstructs or repeatedly interrupts a radio communication service.

2) This device must accept any interference received, including interference that may cause undesired operation. This means that devices using unlicensed spectrum must be designed to coexist in an uncontrolled environment.

Devices utilizing UWB spectrum operate according to similar rules, but they are subject to more stringent requirements because the UWB spectrum underlays other existing licensed and unlicensed spectrum allocations. In order to optimize spectrum use and reduce interference to existing services, the FCCs regulations are very conservative and require very low emitted power.

The UWB spectrum consists of the following three parts:

1) the main spectrum $(3.1-10.6 \mathrm{GHz})$;

2) the lower residual spectrum $(0-3.1 \mathrm{GHz})$;

$3)$ the upper residual spectrum $(10.6-\infty \mathrm{GHz})$.

In theory, the UWB power density within the lower and upper residual spectra should be $(\infty \mathrm{dBm} / \mathrm{MHz})$. In practice, this is not the case; thus, the UWB power density within the residual spectrum should be the minimum possible in order to have very little effect on communications systems working within the residual spectrum of the UWB signal.

The spectrum for the universal mobile telecommunication system (UMTS) lies between 1900-2025 and 2110-2200 MHz. For the satellite service, an own subband in the UMTS spectrum is reserved (uplink 1980-2010 MHz, downlink 2170$2200 \mathrm{MHz}$ ). The remaining spectrum for terrestrial use is divided between two modes of operation. In the frequency division duplex (FDD) mode, there are two equal bands for the uplink (1920-1980 MHz) and for the downlink (2110$2170 \mathrm{MHz}$ ). In the operation, mode time division duplex (TDD) uplink and downlink are not divided by the use of different frequency carriers but by using different timeslots on the same carrier. So, there is no need for a symmetrical spectrum but the remaining unpaired spectrum can be used. The code division multiple access system (CDMA-450) is a Telecommunications Industry Association (TIA)-Energy Information Administration (EIA)-IS-CDMA2000 [CDMA-multicarrier (MC)] system deployed in $450 \mathrm{MHz}$. CDMA-450 provides a larger macrocell size compared to macrocell sizes in other bands (for example, UMTS), which translates to fewer macrocell sites. The CDMA-450 utilized band is the $450-470 \mathrm{MHz}$ band. 
TABLE I

UMTS URBAN MACROCELl DOWNLINK BUDGET

\begin{tabular}{|l|l|l|l|}
\hline A & Maximum link transmit power & $\mathrm{dBm}$ & 43 \\
\hline B & Transmitter gains & $\mathrm{dB}$ & 18 \\
\hline C & Transmitter local losses & $\mathrm{dB}$ & 2 \\
\hline D & Transmitter EIRP & $\mathrm{dB}$ & $\mathrm{A}+\mathrm{B}-\mathrm{C}=59$ \\
\hline E & Receiver noise figure & $\mathrm{dB}$ & 6 \\
\hline F & Thermal noise density & $\mathrm{dBm} / \mathrm{Hz}$ & \\
\hline $\mathrm{G}$ & Noise power & $\mathrm{dBm}$ & $\mathrm{E}+\mathrm{F}^{*} \log 10\left(4 \times 10^{6}\right)$ \\
\hline $\mathrm{H}$ & Load value & 0.5 to 0.95 & \\
\hline $\mathrm{I}$ & Noise rise & $\mathrm{dB}$ & $-10^{*} \log 10(1-\mathrm{H})$ \\
\hline $\mathrm{J}$ & Interference power & $\mathrm{dBm}$ & $10^{*} \log 10\left(10^{\wedge}((\mathrm{G}+\mathrm{I}) / 10)-10^{\wedge}(\mathrm{G} / 10)\right)$ \\
\hline $\mathrm{K}$ & Noise and interference & $\mathrm{dBm}$ & $10^{*} \log 10\left(10^{\wedge}(\mathrm{G} / 10)+10^{\wedge}(\mathrm{J} / 10)\right)$ \\
\hline $\mathrm{L}$ & Processing gain $\left(\mathrm{G}_{\mathrm{p}}\right)$ & $\mathrm{dB}$ & $10^{*} \log 10\left(3.84^{*} 10^{6} / \mathrm{bit} \mathrm{rate}\right)$ \\
\hline M & (E $\left./ \mathrm{N}_{\mathrm{o}}\right)_{\text {req }}$ & $\mathrm{dB}$ & \\
\hline N & Receiver sensitivity & $\mathrm{dBm}$ & $\mathrm{K}+\mathrm{I}-\mathrm{L}+\mathrm{M}$ \\
\hline O & Indoor loss & $\mathrm{dB}$ & 10 \\
\hline P & Maximum path loss & $\mathrm{dB}$ & $\mathrm{D}-\mathrm{I}-\mathrm{N}-\mathrm{O}$ \\
\hline $\mathrm{Q}$ & Log normal fade margin & 8 \\
\hline R & Compensated path-loss & $\mathrm{P}-\mathrm{Q}$ \\
\hline
\end{tabular}

UMTS and CDMA-450 are interference-limited systems, i.e., the multiusers interference is $7-20 \mathrm{~dB}$ higher than the thermal noise of the UMTS or the CDMA-450 receiver.

UMTS and CDMA-450 systems are affected by the UWB interference due to the overlap between the UWB lower residual spectrum and the spectrum of the UMTS or the CDMA-450 systems. Each channel of the UMTS or the CDMA-450 systems is affected by the UWB interfering signal within its band (with a bandwidth of $5 \mathrm{MHz}$ approximately).

Hamalainen et al. studied the coexistence of the UWB system with GSM900, UMTS/wideband CDMA (WCDMA), and GPS [1]. They gave the bit error rate (BER) of the aforementioned systems for different pulse length.

Hamalainen et al. investigated the coexistence of the UWB system with IEEE802.11a and UMTS in modified SalehValenzuela channel [2]. They gave the BER of the UWB system for different types of modulation (direct sequence and time hopping).

Guiliano et al. studied the interference between the UMTS and the UWB system [3]. In [4], Hamalainen et al. investigated the effect of the in-band interference power caused by different kinds of UWB signal at UMTS (WCDMA) frequency bands. The UWB interference was given for the UMTS/WCDMA uplink and downlink. In [5], Hamalainen $e t$ al. studied the effect of the in-band interference power caused by three different kinds of UWB signal on GPS L1 and GSM-900 uplink band.

None of the aforementioned works studied the effect of the UWB interference on the urban macrocell range or capacity.

Deployment of UWB system creates a forbidden zone around the UWB transmitter in which UMTS or CDMA-450 are affected drastically. In practice, the radius of the forbidden zone must be the minimum possible. In our study, we will consider a forbidden zone with $1 \mathrm{~m}$ radius (other value of the forbidden zone radius such as $0.25-2 \mathrm{~m}$ can be considered). Also in our study, we will consider the following acceptable degradation of the UMTS or CDMA-450 systems.

1) The maximum accepted capacity reduction at any moment is $1 \%$.

2) The maximum accepted range reduction at any moment is $1 \%$.

The aim of this paper is to investigate the effect of UWB on UMTS and CDMA-450 urban macrocell downlink performance (range and capacity) when the distance between the UWB transmitter and the mobile receiver under study is $0.1-2 \mathrm{~m}$. Also, we will investigate whether the radiations limits due to the FCC specifications are not enough to drastically affect the UMTS or CDMA-450 performance.

The rest of the paper is organized as follows. In Section II, the methodology for studying the effect of the UWB interference on the UMTS and CDMA-450 downlink performance is presented. In Section III, different results are given. Finally, Section IV addresses the conclusions.

\section{EFFECT OF UWB INTERFERENCE ON THE UMTS AND CDMA-450 DOWNLINK RANGE AND CAPACITY}

The UMTS and the CDMA-450 downlink link-budget that can be used to calculate the absolute range of the downlink adopts the approach given in [6]. The elements to calculate the budget of the UMTS and CDMA-450 downlink are shown in Table I.

For each UMTS or CDMA-450 downlink channel, the UWB interfering signal is due to only $5 \mathrm{MHz}$ of the total UWB spectrum. In this case, we are dealing with only $5 \mathrm{MHz}$ of the total UWB spectrum. To account for UWB interference, an extra source of interference is added linearly to the UMTS and the 
CDMA-450 intrasystem interference. The interference power is calculated by assuming an UWB interfering source at different distances from the UMTS receiver (the mobile station). Therefore, the interference power generated by a device UWB $I_{\mathrm{UWB}}$ (in decibel meters) is given by

$$
I_{\mathrm{UWB}}=P_{\mathrm{UWB}}-L_{\mathrm{UWB}}(d)+G_{\mathrm{UMTS}}
$$

where $P_{\mathrm{UWB}}$ is the UWB effective isotropic radiation power (EIRP) in decibel meters in the UMTS band; $L_{\mathrm{UWB}}(d)$ is the path loss between the UWB device and the UMTS receiver that varies with the separation distance $d$ in meters; and $G_{\mathrm{UMTS}}$ is the UMTS antenna gain.

Given that UWB devices are short-range devices, then the quasi-free space path-loss model with shadowing is often most appropriate especially when the distance between the UWB transmitter and the mobile receiver is less than $7 \mathrm{~m} \mathrm{[7].} \mathrm{Thus,}$ in the UMTS downlink frequency band, the UWB signal propagation loss $L_{\mathrm{UWB}}(d)$ measured in decibels at a distance $d$ from the UWB transmitter is calculated as

$$
\begin{aligned}
L_{\mathrm{UWB}}(d) & \approx 20 \log _{10}\left(\frac{4 \pi}{\lambda}\right)+10 n \log _{10}(d)+\aleph(0, \sigma) \\
& =39.03+10 n \log _{10}(d)+\aleph(0, \sigma)
\end{aligned}
$$

where $\lambda$ is the operating wavelength, $n$ is the indoor propagation exponent (1.6-2.0), and $\aleph(0, \sigma)$ is a Gaussian variable of zero mean and a standard deviation of $\sigma$, representing the deviation from the path-loss mean value (shadowing). Practical value of $\sigma$ is 1.8-3 dB in line-of-sight environment (LOS).

The UMTS signal propagation loss in urban macrocell is given as

$$
L_{\mathrm{UMTS}} \approx L_{\mathrm{o} 1}+10 \gamma \log _{10}\left(R_{\mathrm{km}}\right)
$$

where $L_{\mathrm{O} 1}$ is the UMTS signal propagation loss at a distance of $1 \mathrm{~km}$ including the wall propagation loss $L_{w}$ of (8-12) $\mathrm{dB}$ and $\gamma$ is the UMTS signal propagation exponent (3.5-4.5).

Typical values of $L_{\mathrm{O} 1}$ and $\gamma$ are as follows [6]:

1) $L_{\mathrm{o} 1}=138+L_{w} \mathrm{~dB}$ for the UMTS band;

2) $L_{\mathrm{o} 1}=124+L_{w} \mathrm{~dB}$ for the CDMA-450 band;

3) $\gamma=3.52$.

Equation (3) can be rewritten as

$$
10 \gamma \log _{10}\left(R_{\mathrm{km}}\right)=L_{\mathrm{UMTS}}-L_{\mathrm{o} 1} \text {. }
$$

The term $L_{\mathrm{UMTS}}$ depends on the SNR of the UMTS signal, i.e., lower is the noise, higher is the accepted compensated propagation loss. Thus, we can rewrite (4) as

$$
10 \gamma \log _{10}\left(R_{\mathrm{km}}\right)=k-S-L_{\mathrm{o} 1}
$$

where $k$ is constant; $S$ is the UMTS receiver sensitivity.

When the total noise power consists of the UMTS system noise $I_{\mathrm{UMTS}}$ only, then

$$
10 \gamma \log _{10} R_{\mathrm{UMTS}, \mathrm{o}}=k-S-L_{\mathrm{o} 1}
$$

where $R_{\mathrm{UMTS}, \mathrm{o}}$ is the UMTS initial range.

With the existence of the UWB noise $\left(I_{\mathrm{UWB}}\right)$, the UMTS receiver noise will increase. Thus

$$
10 \gamma \log _{10} R_{\mathrm{UMTS}}=k-S-N-L_{\mathrm{o} 1}
$$

where $R_{\text {UMTS }}$ is the UMTS new range when the UWB affects the UMTS system and $n$ is the UMTS receiver noise increment (in decibels) given by

$$
N=10 \log _{10}\left(\frac{I_{\mathrm{UMTS}}+I_{\mathrm{UWB}}}{I_{\mathrm{UMTS}}}\right) .
$$

Now, from (6) and (7), we have

$$
\begin{aligned}
\left(\frac{R_{\mathrm{UMTS}, \mathrm{o}}}{R_{\mathrm{UMTS}}}\right)^{\gamma} & =10^{\left(\left(k-S-L_{\mathrm{o} 1}\right) / 10\right)-\left(\left(k-S-N-L_{\mathrm{o} 1}\right) / 10\right)} \\
& =10^{N / 10} \\
\left(\frac{R_{\mathrm{UMTS}, \mathrm{o}}}{R_{\mathrm{UMTS}}}\right)^{\gamma} & =\left(\frac{I_{\mathrm{UMTS}}+I_{\mathrm{UWB}}}{I_{\mathrm{UMTS}}}\right) .
\end{aligned}
$$

Thus, the macrocell range $R_{\mathrm{UMTS}}$ with the existence of the UWB interference is given as

$$
R_{\mathrm{UMTS}}=R_{\mathrm{UMTS}, \mathrm{o}} \sqrt[\gamma]{\frac{I_{\mathrm{UMTS}}}{I_{\mathrm{UMTS}}+I_{\mathrm{UWB}}}} .
$$

The normalized range is given as

$$
R_{n}=\frac{R_{\mathrm{UMTS}}}{R_{\mathrm{UMYTS}, \mathrm{o}}}=\sqrt[\gamma]{\frac{I_{\mathrm{UMTS}}}{I_{\mathrm{UMTS}}+I_{\mathrm{UWB}}}} .
$$

Since UMTS is interference-limited system, i.e., its capacity is inversely proportional to the receiver total noise. Then, the capacity $C$ can be written as

$$
C=\frac{A}{I}
$$

where $A$ is constant of proportion and $I$ is the UMTS total interference (thermal noise + multiaccess interference + extra interference). Thus, the normalized capacity $C_{n}$ is given as

$$
C_{n}=\left(\frac{C_{\mathrm{UMTS}}+\mathrm{UWB}}{C_{\mathrm{UMTS}}}\right)=\left(\frac{I_{\mathrm{UMTS}}}{I_{\mathrm{UMTS}}+I_{\mathrm{UWB}}}\right) .
$$

In the frequency band used by CDMA-450, the UWB signal propagation loss in decibels is calculated as

$$
L_{\mathrm{UWB}}(d) \approx 25.7+10 n \log _{10}(d)+\boldsymbol{\aleph}(0, \sigma) .
$$

\section{Results}

In the analysis, we assume that the UWB data rate is higher than the UMTS or CDMA-450 chip rate (higher than $3.84 \mathrm{Mb} / \mathrm{s}$ ). Here, we address the effect of the UWB system on the downlink range and capacity of the UMTS and CDMA-450 systems. We will assume that $\gamma=3.5, n=2$, and $\sigma=3 \mathrm{~dB}$ and that the variable $\aleph$ is truncated at $\pm 3.3 \sigma$.

We will present the results for the following two cases.

1) Average case where the path loss is equal to the mean value of path loss (applicable for only $50 \%$ of the cases).

2) Worst case where the path loss is $3.3 \sigma$ lower than the average value path loss (applicable for $100 \%$ of the cases).

We study the case of voice service $\left(G_{p}=25 \mathrm{~dB}\right.$ and $\left.\left(E_{b} / N_{o}\right)_{\text {req }}=6 \mathrm{~dB}\right)$ assuming an UMTS interference of $-89 \mathrm{dBm}(13 \mathrm{~dB}$ noise rise over the thermal noise due to the UMTS multiusers) and UWB power density of $-60 \mathrm{dBm} / \mathrm{MHz}$ 


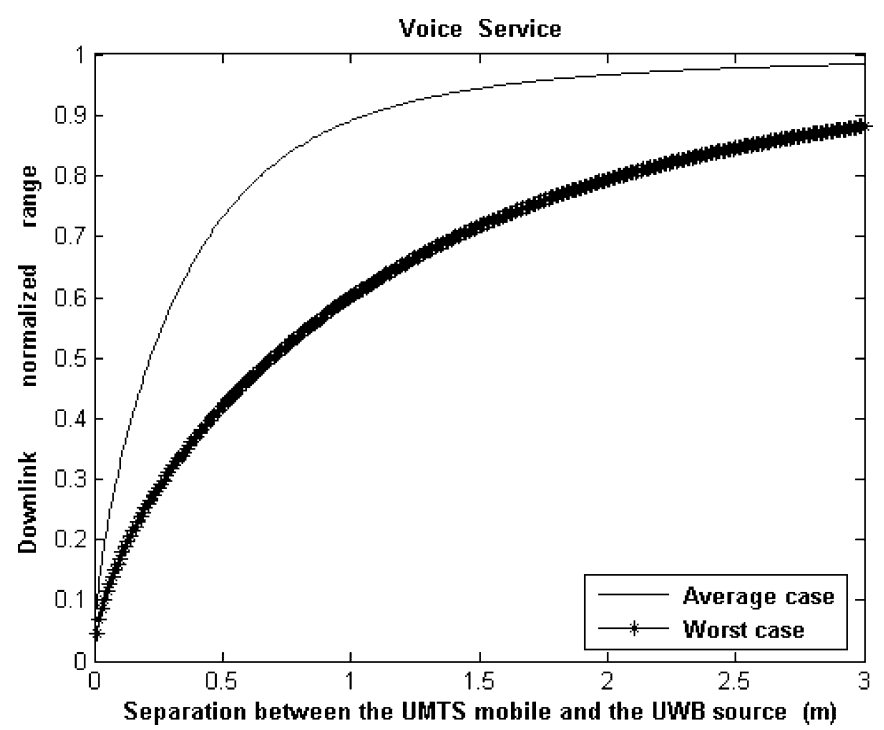

Fig. 1. Effect of the UWB interference on the macrocell range as a function of the separation between the UWB transmitter and the UMTS mobile $\left(P_{\mathrm{UW} \mathrm{B}}=\right.$ $-60 \mathrm{dBm} / \mathrm{MHz}$ ).

(well below the FCC UWB recommended power density of $-51.3 \mathrm{dBm} / \mathrm{MHz}$ at the UMTS downlink frequency band). Fig. 1 shows the downlink macrocell normalized range as a function of the separation between the UMTS mobile and the UWB transmitter. It can be noticed that, for the average case, the UWB signal creates a high interference that reflects a nonacceptable macrocell normalized range reduction of $11 \%$ when the separation is $1 \mathrm{~m}$. For the worst case, the normalized range reduction is $40 \%$ when the separation is $1 \mathrm{~m}$. For higher separation, the interference is lower, and thus, the range reduction is also lower.

Fig. 2 shows the downlink macrocell normalized capacity as a function of the separation between the UMTS mobile and the UWB transmitter. It can be noticed that, for the average case, the UWB signal creates a high interference that gives arise to a nonacceptable macrocell normalized capacity reduction of $33 \%$ when the separation is $1 \mathrm{~m}$. For the worst case, the normalized capacity reduction is $83 \%$ very high to be accepted. For higher separation, the interference is lower, and thus, the normalized capacity reduction is also lower.

Next, we study the case of data service investigating the UWB power density that reduces the UMTS normalized capacity by only $1 \%$ at a forbidden zone with a radius of $1 \mathrm{~m}$. We assume $\left(G p=14.25 \mathrm{~dB}\right.$ that the $\left.\left(E_{b} / N_{o}\right)_{\text {req }}=4.25 \mathrm{~dB}\right)$, UMTS total interference power is $-94.5 \mathrm{dBm}(7.5 \mathrm{~dB}$ noise rise over the thermal noise and thus moderate loaded macrocell) and PUWB power density is $-92.5 \mathrm{dBm} / \mathrm{MHz}$.

Fig. 3 shows the downlink macrocell range as a function of the separation between the UMTS mobile and the UWB transmitter. It can be noticed that, for a separation of $1 \mathrm{~m}$, the UWB signal creates a very low interference. Thus, the normalized range reduction is negligible.

Fig. 4 presents the downlink macrocell capacity as a function of the separation between the UMTS mobile and the UWB transmitter. It can be noticed that, for a separation of $1 \mathrm{~m}$, the UWB

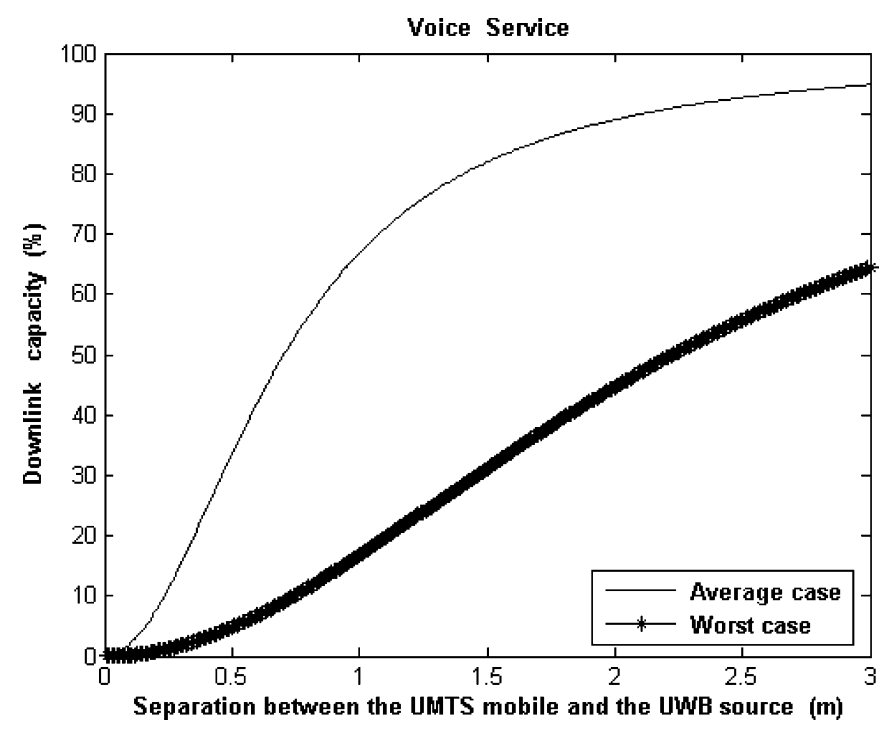

Fig. 2. Effect of the UWB interference on the macrocell normalized capacity as a function of the separation between the UWB transmitter and the UMTS mobile $\left(P_{\mathrm{UW} \mathrm{B}}=-60 \mathrm{dBm} / \mathrm{MHz}\right)$.



Fig. 3. Effect of the UWB interference on the macrocell range as a function of the separation between the UWB transmitter and the UMTS mobile $\left(P_{\mathrm{UW} \mathrm{B}}=\right.$ $-92.5 \mathrm{dBm} / \mathrm{MHz}$ ) and $7.5 \mathrm{~dB}$ noise rise.

signal creates a low interference that provoke a normalized capacity reduction of only $1 \%$ when the worst case is considered.

We have to mention that the performance degradation occurs only when the UWB transmitter is active. Thus, the activity factor of the UWB transmitter and the performance degradation time occurrence have the same percentage.

Then, we study the case of multiple UWB transmitters with one UWB transmitter at each $4 \mathrm{~m} \times 4 \mathrm{~m}$ area of the indoor environment assuming $\mathrm{P}_{\mathrm{UWB}}$ of $-94.5 \mathrm{dBm} / \mathrm{MHz}, 16 \mathrm{UWB}$ transmitters, and an UMTS noise rise of $7.5 \mathrm{~dB}$.

Fig. 5 portrays the downlink macrocell normalized capacity as a function of the UMTS mobile location. It can be noticed that the UWB signal creates a high interference that reflects a high macrocell normalized capacity reduction when the UMTS 


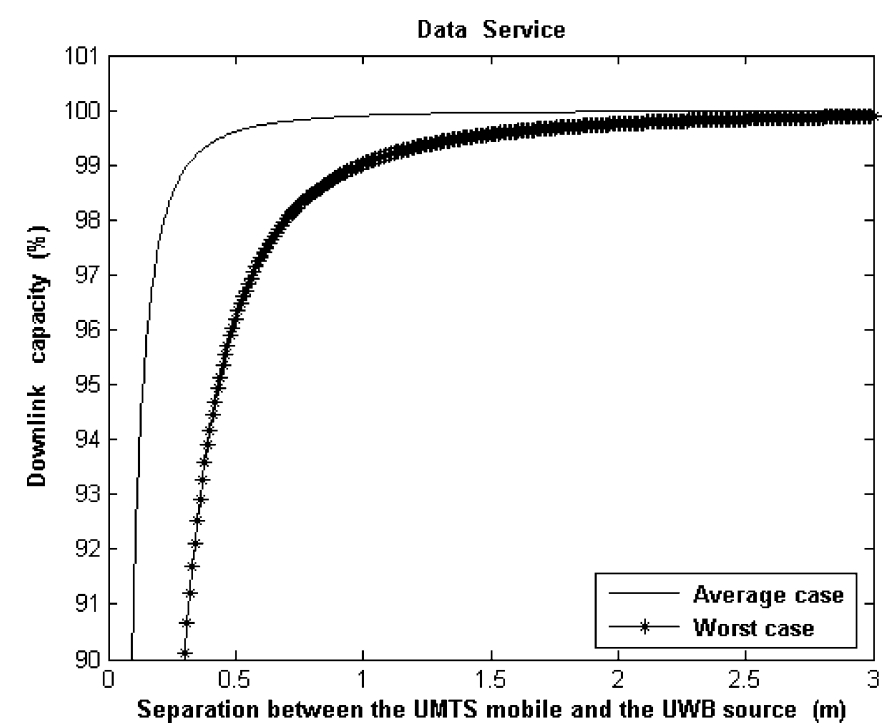

Fig. 4. Effect of the UWB interference on the macrocell normalized capacity as a function of the separation between the UWB transmitter and the UMTS mobile $\left(P_{\mathrm{UW} \mathrm{B}}=-92.5 \mathrm{dBm} / \mathrm{MHz}\right)$ and $7.5 \mathrm{~dB}$ noise rise.

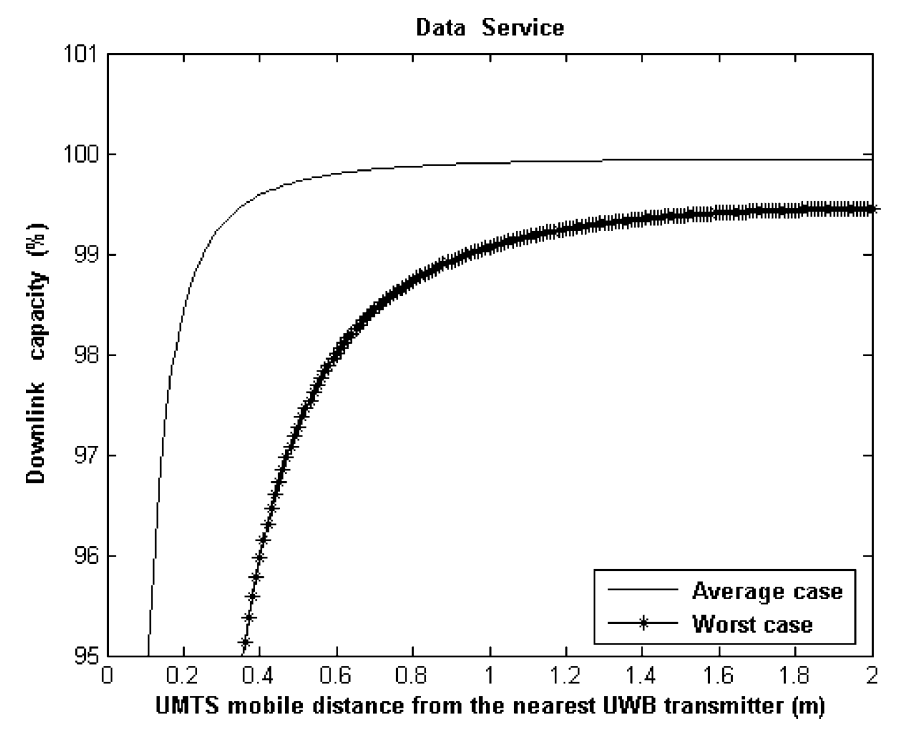

Fig. 5. Effect of the UWB interference on the macrocell normalized capacity as a function of the UMTS mobile distance from the nearest UWB transmitter $\left(P_{\mathrm{UW} \mathrm{B}}=-94.5 \mathrm{dBm} / \mathrm{MHz}\right)$ for multi-UWB transmitters and $7.5 \mathrm{~dB}$ noise rise.

receiver is located at a distance less than $0.4 \mathrm{~m}$ from the nearest UWB transmitter. For a distance greater than $1 \mathrm{~m}$ from the nearest UWB transmitter, the effect of the UWB transmitters is quasi-null (less than $1 \%$ capacity reduction) when we consider the worst case. Result given by Fig. 5 is for the special case when all UWB transmitters have an activity factor of $100 \%$. For lower activity factor (for example, 10\%), the UMTS system can tolerate an UWB power density of $-92.7 \mathrm{dBm} / \mathrm{MHz}$.

It can be concluded that, for the case of single UWB transmitter, the UMTS can easily tolerate the UWB interference when the UWB EIRP is $-92.5 \mathrm{dBm} / \mathrm{MHz}$ for a distance between the UWB transmitter and the UMTS mobile of $1 \mathrm{~m}$ or higher. For the case of multi-UWB transmitter, the UMTS can

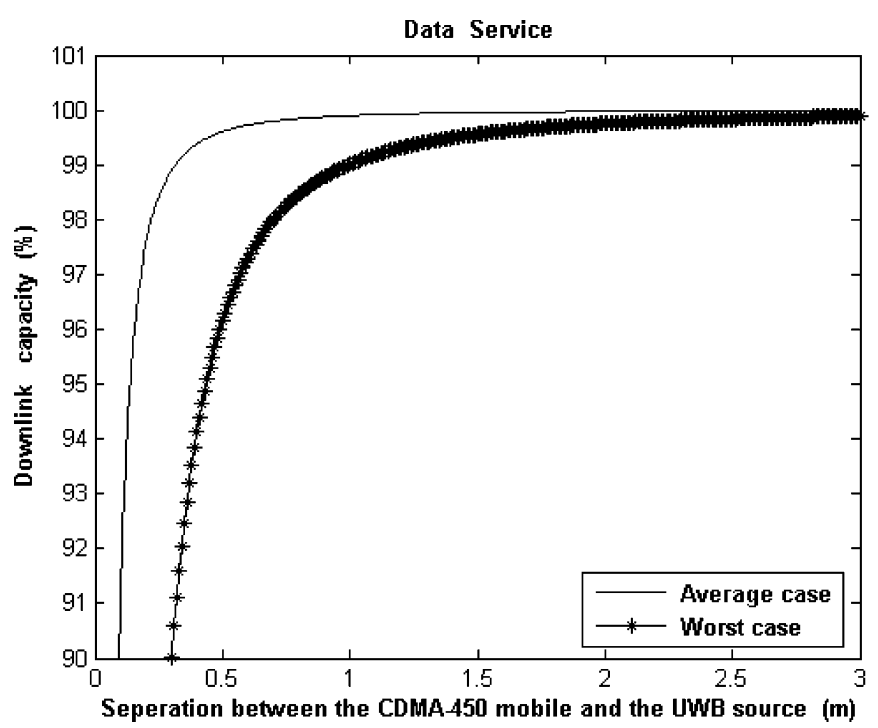

Fig. 6. Effect of the UWB interference on the macrocell normalized capacity as a function of the separation between the UWB transmitter and the CDMA-450 mobile $\left(P_{\mathrm{UW} \mathrm{B}}=-106 \mathrm{dBm} / \mathrm{MHz}\right)$.

easily tolerate the UWB interference when the UWB EIRP is $-94.5 \mathrm{dBm} / \mathrm{MHz}$. The aforementioned numbers are valid for $82 \%$ loaded macrocell.

Next, we study the case of data service $\left(G_{p}=14.2 \mathrm{~dB}\right.$ and $\left.\left(E_{b} / N_{o}\right)_{\text {req }}=4.25 \mathrm{~dB}\right)$ of the CDMA-450 assuming that the CDMA-450 total interference is $-94.5 \mathrm{dBm}(7.5 \mathrm{~dB}$ noise rise over the thermal noise) and UWB power density is $-106 \mathrm{dBm} / \mathrm{MHz}$.

Fig. 6 shows the CDMA-450 downlink macrocell normalized capacity as a function of the separation between the CDMA-450 mobile and the UWB transmitter. It can be noticed that the effect of the UWB interference is very little for a distance greater than $1 \mathrm{~m}$ (lower than $1 \%$ capacity reduction) when we consider the worst case.

Here, we can conclude that, for a single UWB transmitter, the UWB power density that can be tolerated by the CDMA-450 downlink is of the order of $-106 \mathrm{dBm} / \mathrm{MHz}$. For the case of multi-UWB transmitters, the power density that can be tolerated by the downlink of the CDMA-450 system is of the order $-108 \mathrm{dBm} / \mathrm{MHz}$. These numbers are valid for highly loaded macrocells ( $82 \%$ or little more).

If we reduce the forbidden zone radius to $0.5 \mathrm{~m}$, we have to lower the maximum accepted UWB power density by $6 \mathrm{~dB}$. The effect of the UMTS and CDMA-450 signal propagation exponent $(\gamma)$ is very little when it has a value of 3.5-4.5.

From the aforementioned given results we can conclude that the spectrum mask proposed by the FCC for indoor application $(-51.3 \mathrm{dBm} / \mathrm{MHz}$ in the UMTS band and $-41.3 \mathrm{dBm} / \mathrm{MHz}$ for the CDMA-450 band) is very high to be tolerated by the two mobile systems, and we have to have another spectrum mask with lower UWB power density. Thus, the UWB signal with the spectrum shown in Fig. 2 of [8] will drastically affects the UMTS and the CDMA-450 systems performance in spite of its fulfillment of the FCC mask. 


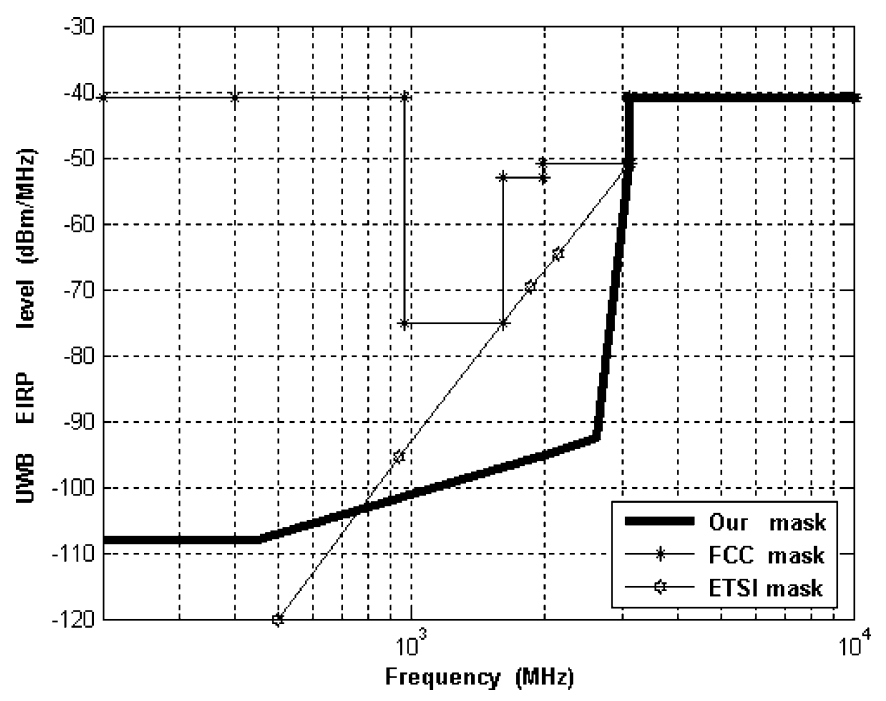

Fig. 7. FCC mask, ETSI mask, and our recommended UWB radiation mask.

From this paper and the FCC regulations, the recommended UWB radiation mask is given in Fig. 7. Fig. 7 also shows the FCC mask and the European Telecommunications Standards Institute (ETSI) mask. For frequencies greater than or equal to $3.1 \mathrm{GHz}$, the three masks have the same values of UWB accepted power density. For frequencies less than $3.1 \mathrm{GHz}$, our recommended mask has always lower accepted UWB power density than the UWB power density given by the FCC recommendations. For frequencies lower than $3.1 \mathrm{GHz}$ and higher than $768 \mathrm{MHz}$, our recommended mask has always lower accepted UWB power density than the UWB power density given by the ETSI mask. For frequencies lower than $768 \mathrm{MHz}$, our recommended mask has always higher accepted UWB power density than the UWB power density given by the ETSI mask. We have to mention that we have taken into account the effect of the UWB on other systems (such as mobile phones at $800 \mathrm{MHz}$ and the proposed UMTS at a frequency of $2600 \mathrm{MHz}$ ) when we have proposed our mask. If we consider the digital television system with receivers that have set-top antennas, then our recommended mask should be the same as the ETSI mask for frequencies lower than $768 \mathrm{MHz}$.

Also, we have to mention that, for an UWB with a bit rate higher than the CDMA chip rate, interference can be considered as Gaussian whatever is the modulation used. It is obvious that the type of modulation used by the UWB system will effect the residual spectrum outside the $3.1-10.6 \mathrm{GHz}$ band. For the multiband [orthogonal frequency division multiplex (OFDM)] UWB system, the power density at a frequency of $2.15 \mathrm{GHz}$ is of the order of $-90 \mathrm{dBm} / \mathrm{MHz}$ while it is of the order of $-95 \mathrm{dBm} / \mathrm{MHz}$ at $0.45 \mathrm{GHz}$. These power densities are higher than our recommended power densities of -94.5 and $-108 \mathrm{dBm} / \mathrm{MHz}$, respectively.

Table II shows the maximum permitted UWB EIRP if other criteria of nonharmful interference is considered assuming forbidden zone with a radius of $1 \mathrm{~m}$. In practice, no one of the service providers accepts a degradation of more than $4 \%$ due to another communication system. Table III shows the maximum
TABLE II

MAXIMUM PERMITTED UWB POWER DENSITY AT UMTS AND CDMA-450 BANDS WITH $1 \mathrm{~m}$ FORBIDDEN ZONE RADIUS

\begin{tabular}{|c|c|c|}
\hline $\begin{array}{c}\text { Maximum accepted } \\
\text { capacity reduction (\%) }\end{array}$ & $\begin{array}{c}\text { UWB power density at } \\
\text { UMTS band }\end{array}$ & $\begin{array}{c}\text { UWB power density at } \\
\text { CDMA-450 band }\end{array}$ \\
\hline $1 \%$ & $-94.5 \mathrm{dBm} / \mathrm{MHz}$ & $-108 \mathrm{dBm} / \mathrm{MHz}$ \\
\hline $2 \%$ & $-91.5 \mathrm{dBm} / \mathrm{MHz}$ & $-105 \mathrm{dBm} / \mathrm{MHz}$ \\
\hline $4 \%$ & $-88.5 \mathrm{dBm} / \mathrm{MHz}$ & $-102 \mathrm{dBm} / \mathrm{MHz}$ \\
\hline $8 \%$ & $-85.5 \mathrm{dBm} / \mathrm{MHz}$ & $-99 \mathrm{dBm} / \mathrm{MHz}$ \\
\hline $16 \%$ & $-82.5 \mathrm{dBm} / \mathrm{MHz}$ & $-96 \mathrm{dBm} / \mathrm{MHz}$ \\
\hline
\end{tabular}

TABLE III

MAXIMUM PERMITTEd UWB POWER DENSITY AT UMTS AND CDMA-450 BANDS WITH 2 m FORBIDDEN ZONE RADIUS

\begin{tabular}{|c|c|c|}
\hline $\begin{array}{c}\text { Maximum accepted } \\
\text { capacity reduction (\%) }\end{array}$ & $\begin{array}{c}\text { UWB power density at } \\
\text { UMTS band }\end{array}$ & $\begin{array}{c}\text { UWB power density at } \\
\text { CDMA-450 band }\end{array}$ \\
\hline $1 \%$ & $-88.5 \mathrm{dBm} / \mathrm{MHz}$ & $-102 \mathrm{dBm} / \mathrm{MHz}$ \\
\hline $2 \%$ & $-85.5 \mathrm{dBm} / \mathrm{MHz}$ & $-99 \mathrm{dBm} / \mathrm{MHz}$ \\
\hline $4 \%$ & $-82.5 \mathrm{dBm} / \mathrm{MHz}$ & $-96 \mathrm{dBm} / \mathrm{MHz}$ \\
\hline $8 \%$ & $-79.5 \mathrm{dBm} / \mathrm{MHz}$ & $-93 \mathrm{dBm} / \mathrm{MHz}$ \\
\hline $16 \%$ & $-76.5 \mathrm{dBm} / \mathrm{MHz}$ & $-90 \mathrm{dBm} / \mathrm{MHz}$ \\
\hline
\end{tabular}

permitted UWB EIRP if other criteria of nonharmful interference is considered assuming forbidden zone with a radius of $2 \mathrm{~m}$. Results of Tables II and III represent the maximum permitted UWB EIRP considering the worst case.

We have to mention that a power density of $-108 \mathrm{dBm} / \mathrm{MHz}$ is difficult to be measured by a conventional spectrum analyzer. Thus, measurements should be done with a resolution bandwidth of $100 \mathrm{kHz}$ searching for a power density of $-98 \mathrm{dBm} / 100 \mathrm{kHz}$ using spectrum analyzer.

\section{CONCLUSION}

The use of the effect of the UWB transmitters on the UMTS downlink for different configuration and environments has been studied. It has been noticed that, for the case of low UWB power density $(-94.5 \mathrm{dBm} / \mathrm{MHz})$, the effect of the UWB signals is low when the distance between the UWB transmitter and the UMTS receiver is greater than $1 \mathrm{~m}$. Also, the effect of the UWB transmitters on the CDMA-450 downlink has been studied. It has been noticed that, for the case of low UWB power density $(-108 \mathrm{dBm} / \mathrm{MHz})$, the effect of the UWB signals is low when the distance between the UWB transmitter and the UMTS receiver is greater than $1 \mathrm{~m}$.

For the case of multi-UWB transmitters, the accepted UWB power density is $1-3 \mathrm{~dB}$ lower than the accepted UWB power density of the UWB single transmitter case. The UWB power density reduction depends on the number of the UWB transmitters and their spatial density, i.e., the higher is the number 
of UWB transmitters and their spatial density, higher should be the reduction of the UWB power density. If we reduce the critical distance to $0.5 \mathrm{~m}$, we have to lower the maximum accepted UWB power density by $6 \mathrm{~dB}$.

\section{REFERENCES}

[1] M. Hamalainen, V. Hovinrn, R. Tesi, J. Iinatti, and M. Latava-aho, "On the UWB system coexistence with GSM900, UMTS/WCDMA, and GPS,” IEEE J. Sel. Areas Commun., vol. 20, no. 9, pp. 1712-1721, Dec. 2002.

[2] M. Hamalainen, R. Tesi, and J. Iinatti, "UWB co-existence with IEEE802.11a and UMTS in modified Saleh-Valenzuela channel," in Proc. Int. Workshop Joint Ultrawideband Syst. Technol. (UWBST 2004) IWUWBS, pp. 45-49.

[3] R. Giuliano, F. Mazzenga, and F. Vatalaro, "On the interference between UMTS and UWB systems," in Proc. IEEE Conf. Ultrawideband Syst. Technol. 2003, pp. 339-343.

[4] M. Hamalainen, V. Hovinen, J. Iinatti, and M. Latva-aho, "In-band interference power caused by different kinds of UWB signals at UMTS/WCDMA frequency bands," in Proc. IEEE Radio Wireless Conf. (RAWCON 2001), Boston, MA, pp. 97-100.

[5] M. Hamalainen, J. Iinatti, V. Hovinen, and M. Latva-Aho, "In-band interference of three kind of UWB signals in GPS L1 band and GSM900 uplink band," in Proc. 12th Int. Symp. Pers., Indoor Mobile Radio Commun. (PIMRC 2001), pp. D76-D80.

[6] H. Holma and A. Toskala, WCDMA for UMTS. New York: Wiley, 2000

[7] A. F. Molisch, K. Balakrishnan, D. Cassioli, Chia-Chin Chong, S. Emami, A. Fort, J. Karedal, J. Kunisch, H. Schantz, and K. Siwiak, "A comprehensive standardized model for ultrawideband propagation channel," IEEE Trans. Antennas Propag., vol. 54, no. 11, pp. 3151-3166, Nov. 2006.

[8] Z. L. Low, J. H. Law, W. T. Ng, and Y. J. Lee, "Pulse detection algorithm for line-of-sight (LOS) UWB ranging applications," IEEE Trans. Antennas Propag. Lett., vol. 4, pp. 63-67, 2005.

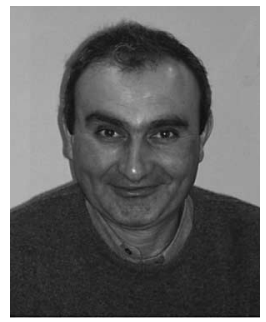

Bazil Taha Ahmed was born in Mosul, Iraq, in 1960. $\mathrm{He}$ received the B.Sc. and M.Sc. degrees in telecommunication engineering from the University of Mosul, Mosul, in 1982 and 1985, respectively, and the Doctor Ingeniero de Telecomunicación degree in WCDMA capacity from the "Escuela Técnica Superior de Ingenieros de Telecomunicación," Universidad Politécnica de Madrid, Madrid, Spain, in 2003.

$\mathrm{He}$ is an Associate Professor at the Universidad Autonoma de Madrid, Madrid. He is the author or coauthor of more than 70 papers published in scientific journals and conference proceedings in the area of the electromagnetic propagation and code division multiple access systems (CDMA), particularly the CDMA capacity. His current research interests include coexistence and wireless access technologies, such as universal mobile telecommunication system (UMTS), worldwide interoperability for microwave access (WiMAX), and ultra-wideband systems and personal area networks (WPANs).

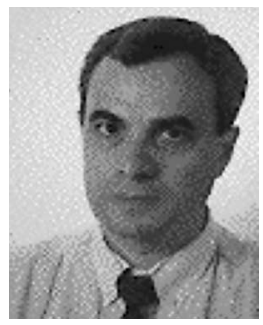

Miguel Calvo Ramon was born in Jaca, Spain, on June 10, 1949. He received the "Ingeniero de Telecomunicación" and the "Doctor Ingeniero de Telecomunicación" degrees in autennas from the "Escuela Técnica Superior de Ingenieros de Telecomunicación," Universidad Politécnica de Madrid, Madrid, Spain, in 1974 and 1979, respectively.

He was a Professor, and later, became a Full Professor in the Department of Signals, Systems and Radiocommunications, Universidad Politecnica de Madrid, where he is currently with the European Telecommunications Standards Institute (ETSI). His current research interests include coexistence and wireless access technologies, such as universal mobile telecommunication systems (UMTS), worldwide interoperability for microwave access (WiMAX), and ultra-wideband systems and personal area networks (WPANs). 